\title{
PIWIL2 promotes progression of non-small cell lung cancer by inducing CDK2 and Cyclin A expression
}

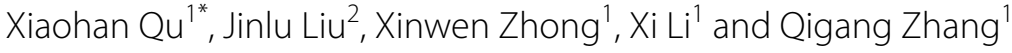

\begin{abstract}
Background: PIWI proteins have important roles in tumorigenesis due to their interaction with piRNAs. Recent studies suggest that PIWI proteins affect prognosis of various cancers.

Methods: In the present study, PIWI genes expression was assayed in non-small cell lung cancer (NSCLC). To determine the effects of PIWIL2 on NSCLC cells, overexpression and interference assays were performed using the A549 and $\mathrm{H} 460$ cell lines. The tumor formation model was performed to demonstrate the effects of PIWIL2 on tumor formation in vivo.

Results: PIWIL2 was increased both at the RNA and protein level in malignant cancer tissues compared with adjacent normal tissue. Moreover, increased PIWIL2 gene expression was negatively correlated with prognosis in NSCLC patients. Overexpression and interference of PIWIL2 promoted and depressed cell proliferation, respectively. Meanwhile, PIWIL2 interference arrested cells at the G2/M stage. In addition, we found that CDK2 and Cyclin A expression were correlated with PIWIL2 expression. Moreover, transfection of PIWIL2 promoted tumor growth in nude mice.
\end{abstract}

Conclusion: Our findings shed light on the function of PIWIL2 in NSCLC and suggest potential prognostic and therapeutic value.

Keywords: PIWIL2, Non-small cell lung cancer, Cyclin A, CDK2, Prognosis

\section{Background}

Non-small cell lung cancer (NSCLC) is one of the most common cancers worldwide and is associated with high mortality rates [1]. Several biomarkers have been used to detect progression have been used in diagnosis of NSCLC mortality. However, both NSCLC-specific antigens and mRNA-based biomarkers are associated with overdiagnosis and overtreatment [2]: mRNA-based biomarkers are seldom specific to NSCLC tissue and are commonly expressed by epithelial cells, including normal, hyperplastic and cancerous cells [2]; meanwhile, NSCLC-specific antigen serum levels could reflect cancer progression or NSCLC hyperplasia, infection or inflammation [3]. Thus, there is a need for improved NSCLC

\footnotetext{
*Correspondence: han_seal@163.com

${ }^{1}$ The First Affiliated Hospital, China Medical University, NO. 155, Nanjing

North Street, Heping District, Shenyang 110001, Liaoning, China

Full list of author information is available at the end of the article
}

biomarkers. Meanwhile, the most common therapeutic strategy for NSCLC, including exairesis, chemotherapy and radiotherapy, are associated with poor prognosis and low long-term survival rates [4]. New strategies such as gene therapy and stem cell therapy have been used in clinical treatment $[5,6]$. In addition, epigenetic research presents another potential cancer therapeutic avenue; understanding epigenetic changes during NSCLC progression could contribute to diagnosis, predicting prognosis and exploring new treatment strategies $[7,8]$.

Chromosomal instability is a common feature of cancer cells that often displays deletions, rearrangements and duplications of different DNA fragments in the genome [9]. Specific alterations in miRNA expression, methylation levels and histone modifications are all regarded as causative factors in the etiology of cancer $[10,11]$. Indeed, aberrant DNA methylation has been found in transposable elements (TEs)-regions that may 
be associated with cancer progression [12]; erratic TEs could lead to genomic instability and affect expression levels of both oncogenes and tumor suppressor genes [13, 14]. Hyperactive TEs therefore appear to play an integral role in cancer development. PIWI proteins are key factors in controlling TE stability $[15,16]$. By interacting with PIWI-interacting RNAs (piRNAs), PIWI protects the integrity and stability of the genome from silencing TEs [17]. Much progress has been made in understanding the molecular function of PIWI in several cancers; however, the role of PIWI in NSCLC remains unknown.

Although the PIWI-piRNA pathway was first identified in the testis, PIWI has subsequently been found to be highly expressed in many different types of cancer [18]. PIWI is expressed in breast cancer [19] and gastrointestinal cancer $[20,21]$ but not in the corresponding normal tissues. PIWI homologs are common in vertebrates; for example, in mice, three different homologs of PIWI were found [22] while in humans, PIWIL1, PIWIL2, PIWIL3 and PIWIL4 were identified [23, 24]. These homologs may have different functions depending on the type of cancer. PIWIL1 promotes cancer growth and is associated with increased mortality $[20,25]$; increased levels of PIWIL2 have been reported in breast $[26,27]$ and cervical cancer [28]; and increased levels of PIWIL3 and PIWIL4 have been reported in colon cancer [29]. To date, PIWI gene expression has not been investigated in NSCLC; the expression patterns and function of PIWI in NSCLC therefore warrants investigation.

In the present study, we focus on the expression pattern of PIWI homologs in the context of NSCLC progression and prognosis. We investigated the function of PIWI in a NSCLC cell line, using cell transfection. The biological activities of the NSCLC cell line were confirmed after PIWI over-expression and silencing. Finally, Cyclins and Cyclin-dependent kinases (CDKs) were detected after PIWI over-expression and silencing to investigate the mechanism of PIWI on NSCLC cell progression both in vivo and in vitro. This study provided novel insights into the role of PIWI in NSCLC that suggest potential diagnostic, prognostic and therapeutic value for PIWI in NSCLC.

\section{Methods}

Materials

NSCLC samples were provided by the China Medical University (Shenyang, China) in accordance with the guidelines of the China Medical University of Medicine Research Ethics Committee. All patients provided informed consent. NSCLC tissues and adjacent tissues were collected from patients who underwent resection. Tumor tissues were confirmed as such by haematoxylin and eosin staining. Only those samples with more than
$70 \%$ tumor content were used for further study as tumor tissues.

\section{Cell culture and transfection}

The human NSCLC cell line, A549 and H460 were obtained from the Shanghai Cell Bank at the Chinese Academy of Sciences. The cells were cultured in Dulbecco's modified Eagle's medium (DMEM) (Gibco, Gaithersburg, Maryland, USA) containing $10 \%$ fetal bovine serum (Gibco) at $37^{\circ} \mathrm{C}$ in a incubator at $5 \% \mathrm{CO}_{2}$.

The pcDNA3.1+ vector containing c-Myc-tagged PIWIL2 was constructed in our laboratory as previously described [30]. The c-Myc promoter was synthesized by Sangon Biotech (Shanghai, China). The PIWI fragment was amplified from human iPS cell lines with the primer set: $5^{\prime}$-TTC TCG AGA TGG ATC CTT TCC GAC CAT C- $3^{\prime}$ and $5^{\prime}$-TTC CAT GGT CAC AGG AAG AAC AGG TTC TC- $3^{\prime}$. The primer sets were designed to contain the full length of coding sequence. Amplified PIWI cDNA was digested with NcoI and Xhol (New England BioLabs [NEB], Hertfordshire, UK). The digested fragment was then subcloned between the NcoI site and the XhoI site of the pcDNA3.1+ vector. The construction of shRNA for PIWIL2 was previously reported [30] (PIWIL2 shRNA1: 5'-ACC GGC CUG GGU UGA ACU AAA-3', PIWIL2 shRNA2: $5^{\prime}$-ACA GAA UCA AAC ACU GUG A-3'). Transfection was performed in 6-well plates with Lipofectamine 2000 (Invitrogen, Carlsbad, CA, USA) according to the manufacturers' protocol. Cells were then replated at a density of $2 \times 10^{5}$ cells per well and cultured for $48 \mathrm{~h}$ after which they were assayed by realtime PCR, western blot and immunofluorescence staining. For rescue groups, after $24 \mathrm{~h}$ post interference or over-expression transfection, the second over-expression transfection or interference were performed, respectively. Then the cells were analyzed at $48 \mathrm{~h}$ post the second transfection.

\section{MTT assays}

To assay the cell proliferation rate, 24-well plates at a density of $2 \times 10^{4}$ cells/well were seeded after transfection. Subsequently, the cells were analyzed by methyle thiazol tetrazolium assay (MTT assay, Sigma-Aldrich, St. Louis, MN, USA). The amount of MTT formazon product was determined by using a microplate reader and the absorbance was measured at $570 \mathrm{~nm}$ (Berthold, Tokyo, Japan).

\section{Real-time PCR}

Total RNAs were extracted from tissues or cells using Trizol (Invitrogen, Carlsbad, CA, USA). The quality and quantity of total RNA was determined by agarose gel electrophoresis and by the BioPhotometer Plus (Eppendorf AG, Hamburg, Germany). For reverse transcription, 
$1 \mu \mathrm{g}$ total RNA was transcribed for each sample to complementary DNA (cDNA) using M-MLV reverse transcriptase (Invitrogen) according to the manufacturer's protocol. The primers for real-time PCR are shown in Table 1. The PCR conditions were as follows: $95{ }^{\circ} \mathrm{C}$ for $3 \mathrm{~min}, 40$ cycles at $95{ }^{\circ} \mathrm{C}$ for $12 \mathrm{~s}$ and $55{ }^{\circ} \mathrm{C}$ for $40 \mathrm{~s}$. GAPDH was used as an internal PCR control. Meanwhile, a no-template control and melt curve analysis were used to monitor contamination. The relative expression levels were calculated using the $2^{-\Delta \Delta \mathrm{Ct}}$ method.

\section{Western blot}

The levels of PIWI proteins, CDK proteins, Cyclin proteins and GAPDH were determined by western blot. Primary rabbit polyclonal antibodies against PIWIs (PIWIL1, SAB1300682; PIWIL2, SAB2105190; PIWIL3, SAB4200150; PIWIL4, SAB2105885), CDKs (CDK1, SAB4500050; CDK2, SAB4503706; CDK4, SAB4300695), Cyclins (Cyclin B, SAB4503501; Cyclin A, SAB4503499; Cyclin D1, SAB4502603) and GAPDH (SAB2100894) were purchased from Sigma-Aldrich (St. Louis, MO, USA). Secondary antibodies were HRP-conjugated anti-rabbit IgG (Sigma-Aldrich). Both tissues and cells were homogenized using RIPA buffer (50 mM Tris pH 8, $150 \mathrm{mM} \mathrm{NaCl}, 1 \%$ NP-40, $0.5 \%$ DOC, $0.1 \%$ SDS, $1 \mathrm{mM}$ DTT, protease and phosphatase inhibitors). Extracted proteins were quantified using the BCA protein assay kit from Sangon Biotech (Shanghai, China). For each sample, $15 \mu \mathrm{g}$ protein were electrophoresed on a $12 \%$ sodium dodecyl sulfate polyacrylamide gel. The sample was next transferred onto polyvinylidene fluoride membranes (Millipore, Billerica, MA, USA). Proteins were blocked at room temperature for $1 \mathrm{~h}$ using $4 \%$ skim milk and incubated with each antibody

Table 1 Primers used in the present study

\begin{tabular}{|c|c|c|}
\hline & Forward $5^{\prime}-3^{\prime}$ & Reverse $5^{\prime}-3^{\prime}$ \\
\hline PIWIL1 & $\begin{array}{l}\text { TGTCTGTTGTCAAGTAATCG- } \\
\text { GAAGG }\end{array}$ & TTGCTGTTTGCCTAAGGTTCG \\
\hline PIWIL2 & TACCTTCAGCACACCGTCC & GACACTGTATITTGACGAGGT \\
\hline PIWIL3 & $\begin{array}{l}\text { GAGCCCAGATACAGTACA- } \\
\text { GCGTT }\end{array}$ & GGACTGCCCCACGAGGTAA \\
\hline PIWIL4 & TACTGTATCGGACCTGAATCA & TTCAGCCACAGCCTTCATCAG \\
\hline CDK1 & $\begin{array}{l}\text { TGCTAAGTTCAAGTTTCGTA } \\
\text { ATGCT }\end{array}$ & $\begin{array}{l}\text { AAGGACTGAGATGATTTAAGC- } \\
\text { CAAC }\end{array}$ \\
\hline CDK2 & $\begin{array}{l}\text { ATCCGCCTGGACACTGAG } \\
\text { ACT }\end{array}$ & TGGAGGACCCGATGAGAATG \\
\hline CDK4 & $\begin{array}{l}\text { CACAGTTCGTGAGGTGGC } \\
\text { TTTA }\end{array}$ & $\begin{array}{l}\text { TGTCCTTAGGTCCTGGTCTA- } \\
\text { CATG }\end{array}$ \\
\hline Cyclin B & TTGGTTTCTGCTGGGTGTAGG & CCATGTTGATCTTCGCCTTATTT \\
\hline Cyclin A & GCATGTCACCGTTCCTCCTTG & GGGCATCTTCACGCTCTATTTT \\
\hline Cyclin D1 & TCGCTGGAGCCCGTGAA & CCGCCTCTGGCATTTTGG \\
\hline GAPDH & CGCTCTCTGCTCCTCCTGTT & CCATGGTGTCTGAGCGATGT \\
\hline
\end{tabular}

(for PIWIs, 1:1000; CDKs, 1:1000; Cyclins, 1:100; GAPDH, 1:2000) at $4{ }^{\circ} \mathrm{C}$ overnight. After three washes with TBST buffer (pH 7.6, $20 \mathrm{mM}$ Tris-HCl, $137 \mathrm{mM} \mathrm{NaCl}, 0.01 \%$ Tween-20), membranes were incubated with HRP-conjugated anti-rabbit IgG and visualized using enhanced chemiluminescence (ECL, Millipore, Billerica, MA, USA).

\section{Immunohistochemical staining (IHC)}

Tissue samples were fixed with $10 \%$ buffered formalin and $6-\mu \mathrm{m}$-thick tissue sections were cut. The sections were then incubated with monoclonal antibody (for PIWIs, 1:100; CDKs, 1:200; Cyclins, 1:200) and the same secondary antibody that was used for western blotting. Horseradish peroxidase streptavidin complex (Beyotime, Wuhan, China) was used to visualize the signals. Sections were color-developed with diaminobenzidine and stained with hematoxylin (Beyotime).

\section{Immunofluorescence microscopy}

Protein expression was measured by immunofluorescence, using monoclonal antibodies (for CDKs, 1:100; Cyclins, 1:100) that were detected by FITC-conjugated goat anti-rabbit IgG (Sigma-Aldrich) at a dilution of 1:200. DAPI $(1 \mu \mathrm{g} / \mathrm{ml})$ was used to stain nuclei.

\section{Flow cytometry}

Cell activities and apoptosis were measured by flow cytometry after over-expression and inhibition of PIWIL2 in NSCLC cells. Annexin V-FITC and propidium iodide (PI) (BioVision, Milpitas, CA, USA) staining was used according to the manufacturer's instructions. For each sample, at least 30,000 cells were analyzed. Experiments were performed in triplicate.

\section{Tumor formation in a nude mouse model}

Five-week-old nude mice were used to confirm the effect of PIWIL2 on tumor formation. The experiment was approved by the ethics committee of the China Medical University (Shenyang, China). Mice were randomly divided into two groups of ten $(n=5)$. One group was treated with A549 cells while other was treated with A549 cells containing PIWIL2 expression vectors; 30,000 A549 cells (in $1 \mathrm{ml}$ DMEM) were injected every 3 days for 21 days on the dorsal side of the mouse. Tumor volumes were calculated every 3 days according to the following the formula: Volume $=$ length $\times$ width $\times$ height $\times 3.14 / 6$. At 21 days, tumor tissues were assayed by real-time PCR, western blot and IHC.

\section{Statistical analysis}

Real-time PCR, MTT assays and apoptosis rates are presented as mean \pm standard deviation (SD). One-wayANOVA analysis was used to analyze differences among 
groups and results where $\mathrm{P}<0.05$ were considered significant. Survival curves were plotted according to the Kaplan-Meier method. Of the 126 patients involved in this study, 62 expressed relatively low levels of PIWIL2 (fold-change relative expression to adjacent tissue was between 2 and 4) while 64 expressed high levels of PIWIL2 (fold-change relative expression to adjacent tissue was between 5 and 7) in cancer tissues, as judged by realtime PCR (Table 2). Multivariate analysis was conducted using Cox's proportional hazards regression model. Patients' survival information was collected telephonically. Tumor stage was classified based on the 2011 Union for International Cancer Control (UICC) TNM classification of malignant tumors. The nuclear grading was performed according to Fuhrman's system [31]. All data were analyzed using SPSS 17.0 (SPSS Inc., Chicago, IL, USA).

\section{Results}

PIWIL2 expression is elevated in NSCLC tissues

To demonstrate the roles of PIWI in NSCLC, real-time PCR, western blot and IHC were employed to assay the

Table 2 Clinical characteristics of patients with liver cancer in the present study

\begin{tabular}{|c|c|c|c|c|c|}
\hline \multirow[t]{2}{*}{ Parameters } & \multirow[t]{2}{*}{$\mathrm{n}$} & \multicolumn{2}{|c|}{ PIWIL2 } & \multirow[t]{2}{*}{$x^{2}$} & \multirow[t]{2}{*}{$P$ value } \\
\hline & & High & Low & & \\
\hline Total & 126 & 64 & 62 & 0.189 & 0.87 \\
\hline \multicolumn{6}{|l|}{ Sex } \\
\hline Male & 60 & 30 & 30 & & \\
\hline Female & 66 & 34 & 32 & & \\
\hline Age (years) & & & & 1.56 & 0.56 \\
\hline$\geq 50$ & 67 & 34 & 33 & & \\
\hline$<50$ & 59 & 30 & 29 & & \\
\hline T stage & & & & 6.15 & 0.03 \\
\hline $\mathrm{T} 1$ & 75 & 40 & 35 & & \\
\hline $\mathrm{T} 2$ & 32 & 18 & 14 & & \\
\hline $\mathrm{T} 3 / 4$ & 19 & 6 & 13 & & \\
\hline N stage & & & & 4.62 & 0.05 \\
\hline No & 96 & 44 & 52 & & \\
\hline $\mathrm{N}+$ & 30 & 20 & 10 & & \\
\hline Metastasis & & & & 5.69 & 0.03 \\
\hline No (M0) & 72 & 35 & 37 & & \\
\hline Yes (M1) & 54 & 29 & 25 & & \\
\hline Recurrence & & & & 7.62 & 0.02 \\
\hline No & 70 & 34 & 36 & & \\
\hline Yes & 56 & 30 & 26 & & \\
\hline Fuhrman & & & & 11.52 & 0.02 \\
\hline 1 & 21 & 11 & 10 & & \\
\hline 2 & 64 & 30 & 34 & & \\
\hline 3 & 22 & 11 & 11 & & \\
\hline 4 & 19 & 12 & 7 & & \\
\hline
\end{tabular}

expression levels of the four PIWI homologs (PIWIL1, PIWIL2, PIWIL3 and PIWIL4) in NSCLC tissues and adjacent tissues. PIWIL2 expression was significantly higher, both at the mRNA $(\mathrm{P}<0.05)$ (Fig. 1a) and protein levels (Fig. 1b, c), in malignant cancer tissues compared to adjacent tissues. No significant difference was observed in other PIWI genes (PIWIL1, PIWIL3 or PIWIL4) between NSCLC tissues and adjacent tissues (Fig. 1).

\section{PIWIL2 is associated with poor prognosis in NSCLC}

We observed 126 patients for a period of 100 months to determine the relationship between PIWIL2 expression and NSCLC prognosis. Patients were divided into two groups: those that had high levels of PIWIL2 expression (mRNA expression levels higher than 5), and those with low levels of PIWIL2 expression (mRNA expression levels lower than 4). Overall survival and disease-free survival were estimated. We found a significant negative correlation between PIWIL2 and overall survival as well as disease-free survival (Fig. 1d, e).

\section{PIWIL2 regulates NSCLC cell progression}

To determine the role of PIWIL2 on NSCLC cells, we used RNA interference and overexpression vectors to control PIWIL2 expression. As expected, the overexpression vectors increased, while the interference assay decreased PIWIL2 levels, as confirmed by real-time PCR (Fig. 2A) and western blot (Fig. 2B). Both shRNA1 and shRNA2 depressed expression of PIWIL2 which confirmed no off target effects in the present experiment (Fig. 2A, B).

The MTT assays indicated that cell proliferation could be depressed by both shRNA1 and shRNA2 interference while overexpression of PIWIL2 induced cell proliferation both in A549 and H460 (Fig. 3A, B). Using flow cytometry, we found that the interference group had higher levels of apoptosis compared with the overexpression group (Fig. 3C, D). Furthermore, in the interference group, more cells arrested at the G2/M stage compared with the other groups (Fig. 3C, E).

\section{PIWIL2 regulates CDK2 and Cyclin A expression in NSCLC cells}

We next investigated the mechanism whereby PIWIL2 causes $\mathrm{G} 2 / \mathrm{M}$ cell cycle arrest by measuring the expression of Cyclins and CDKs. The interference of PIWIL2 was used shRNA1 which has been proved with no off target effects. The A549 cell line was used to determined the gene expression. The present result indicated that no significant differences were observed for CDK1, CDK4, Cyclin B or Cyclin D at mRNA or at the protein level (Fig. 4A-C). On the contrary, CDK2 and Cyclin A 

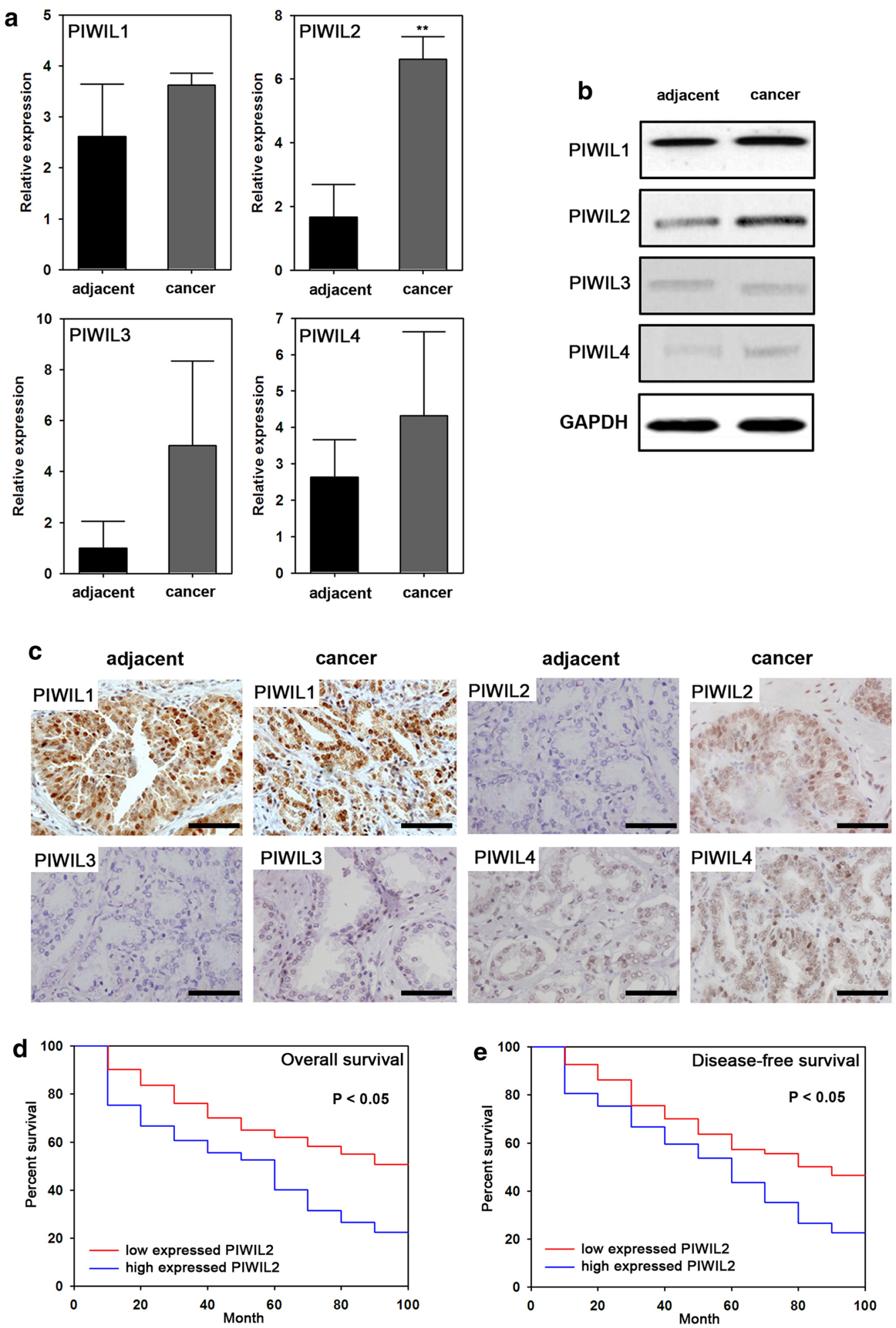

Fig. 1 Expression of PIWIL2 in NSCLC and multivariate Cox's regression analyses of primary NSCLC patients $(n=126)$. Real-time PCR (a), western blot (b) and IHC (c) analysis of adjacent and NSCLC tissues. d Overall survival rates and its correlation with PIWIL2 expression. The cumulative overall survival rate for patients in the PIWIL2-high group was significantly lower than that for patients in the PIWIL2-low group $\left(\chi^{2}=7.54 ; P=0.03\right)$. e Disease-free survival rates and its correlation with PIWIL2 expression. The cumulative disease-free survival rate for patients in the PIWIL2-high group was significantly lower than that for patients in the PIWIL2-low group $\left(\chi^{2}=8.78 ; P=0.02\right)$. Real-time PCR data are presented as the mean \pm SD $(n=30) ;{ }^{* * P}<0.01$; for $\mathrm{IHC}$, scale bars $=100 \mu \mathrm{m}$. The longest follow-up time was 100 months 

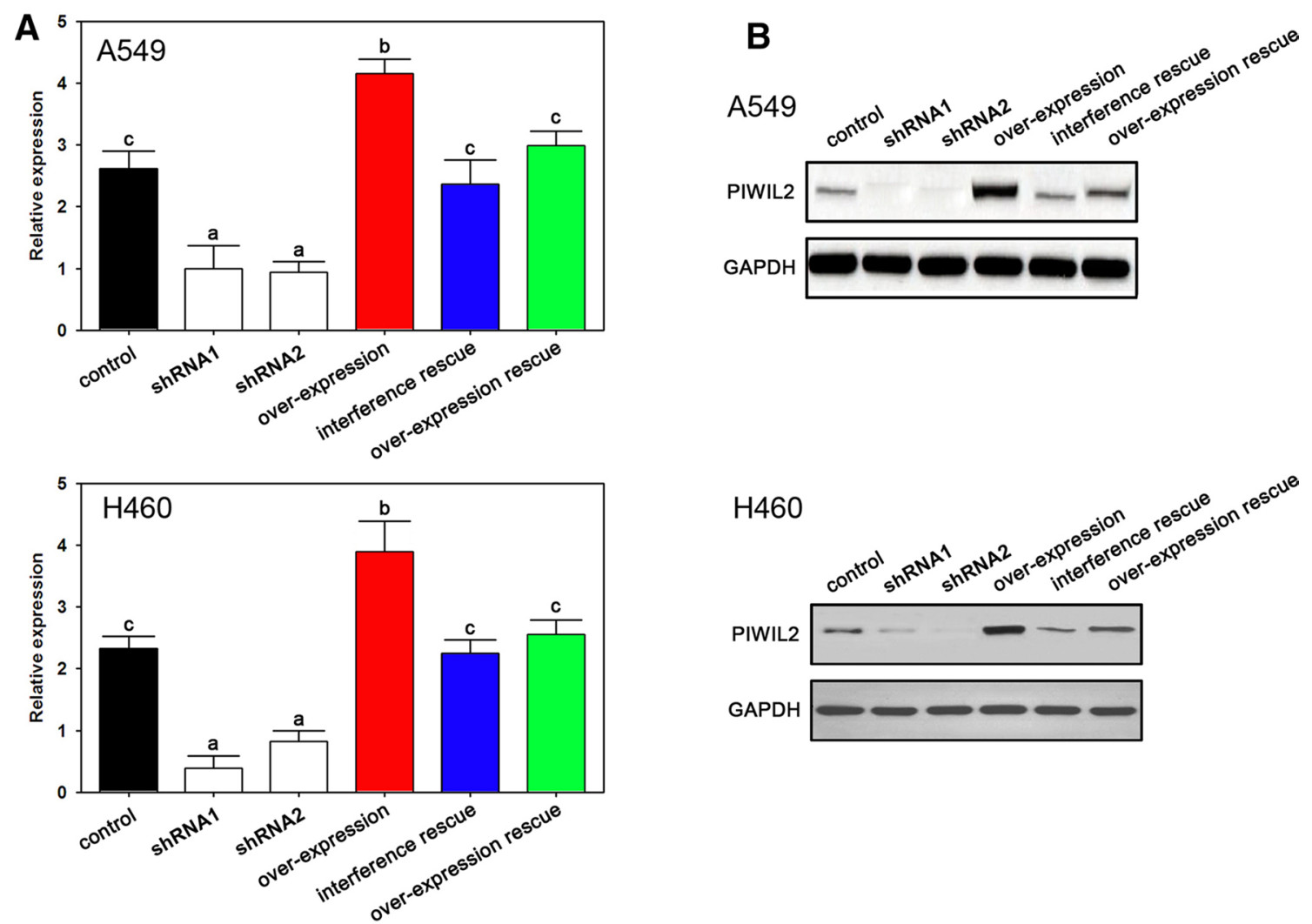

Fig. 2 The expression of PIWIL2 in NSCLC cells after transfection. Real-time PCR (A) and western blot (B) analysis indicated that mRNA and protein expression were down-regulated and up-regulated by interference and over-expression of PIWIL2, respectively

expression were significantly decreased or increased following PIWIL2 interference, or overexpression, respectively (Fig. 4A-C).

\section{PIWIL2 promotes tumor growth in vivo}

We found that mice treated with PIWIL2-transfected A549 cells had significantly increased tumor volumes compared to controls (Fig. 5a). Moreover, PIWIL2 expression promoted expression of CDK2 and Cyclin A both at mRNA and protein level (Fig. 5b-d), which indicates that PIWIL2 induces CDK2 and Cyclin A expression and promotes tumorigenesis in nude mice.

\section{Discussion}

In this study, we explored PIWI expression in NSCLC as well as its role in tumor progression. By comparing the expression of the four PIWI homologs (PIWIL1, PIWIL2, PIWIL3 and PIWIL4), we showed that only PIWIL2 was highly expressed in malignant NSCLC tissues compared with adjacent tissues. These results suggested that PIWIL2 may play a crucial role in the progression of NSCLC. Moreover, high levels of PIWIL2 expression were associated with decreased overall survival and disease-free survival rates. Similar results have been demonstrated in breast [27] and endometrial cancer [28]. Thus, in cancers, the overexpression of PIWIL2 is key evidence in the malignant and pernicious [30]. However, PIWI expression patterns vary by cancer type. For instance, all PIWI proteins are overexpressed in colon cancer [29], while only PIWIL2 is expressed in breast cancer [26]. Although PIWI genes are potentially useful diagnostic and prognostic biomarkers, the heterogeneous expression patterns between different cancer types is still not well understood. We showed that PIWIL2 is highly expressed in NSCLC and is a potential indicator of NSCLC prognosis.

Once we confirmed the aberrant expression of PIWIL2 in NSCLC, we investigated the potential role of PIWIL2 in NSCLC progression in NSCLC cells using overexpression and interference of PIWIL2. High PIWIL2 expression significantly promoted cell proliferation and activities. Conversely, inhibition of PIWIL2 triggered apoptosis and G2/M cell cycle arrest. It is widely recognized that PIWIL2, as a small RNA binding protein, mediates genome stability and regulates genes expression [32, 33]. The PIWI-piRNA pathway was first illustrated 

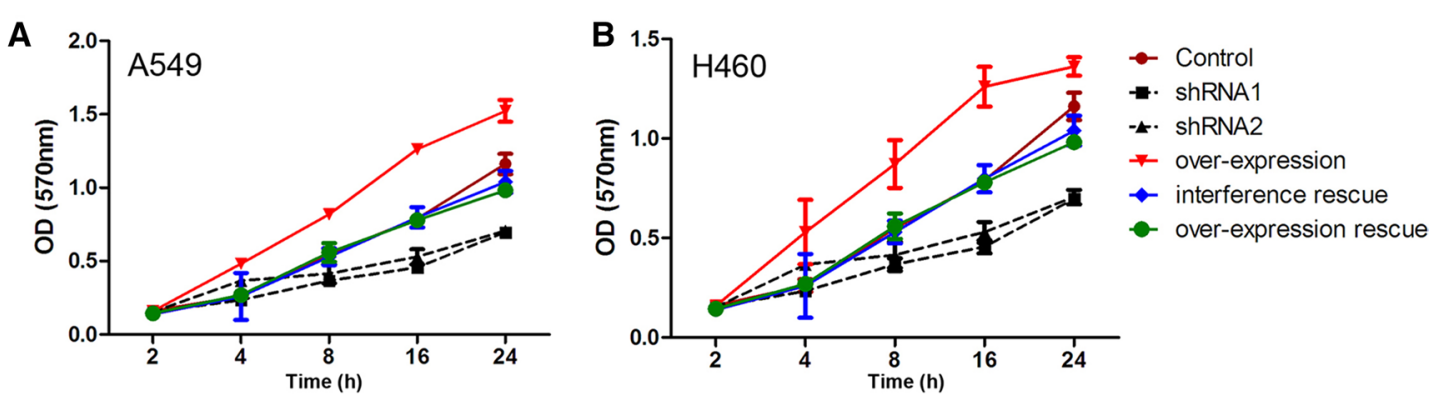

C
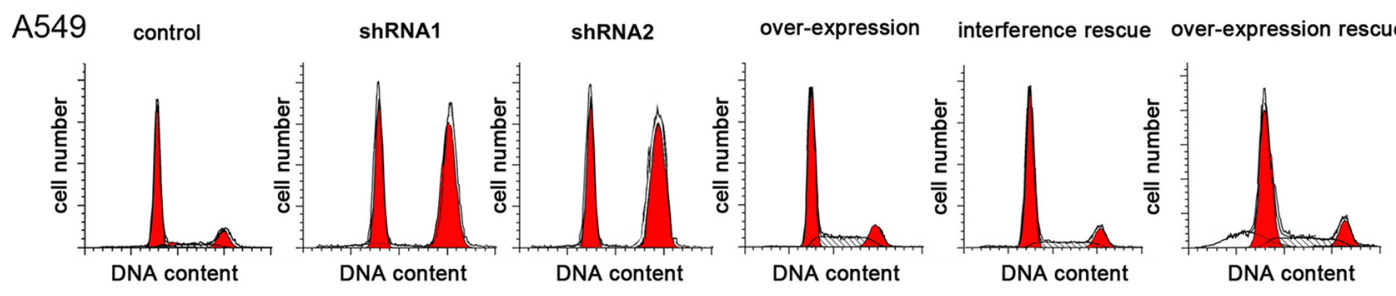

H460 control
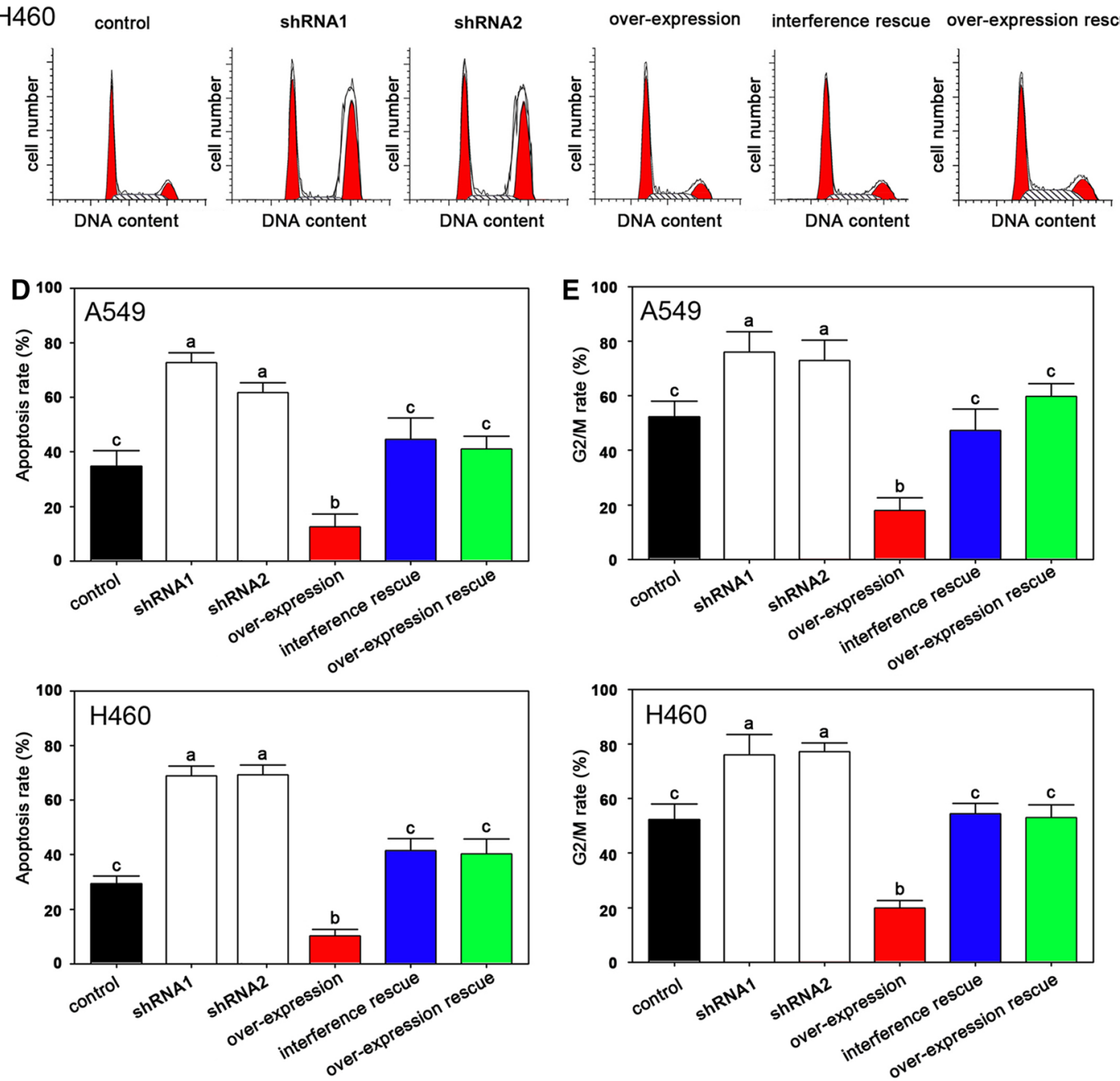

Fig. 3 Effects of PIWIL2 on NSCLC cells after transfection. A and B indicate the cell proliferation after transfection by MTT in A549 and H460. C Flow cytometry analysis. D Apoptosis rates of PIWIL2 interference and over-expression groups, determined by flow cytometry analysis. E G2/M stage arrested rate was increased after repression of PIWIL2. Different lower case characters represent significant differences, $P<0.05$ 

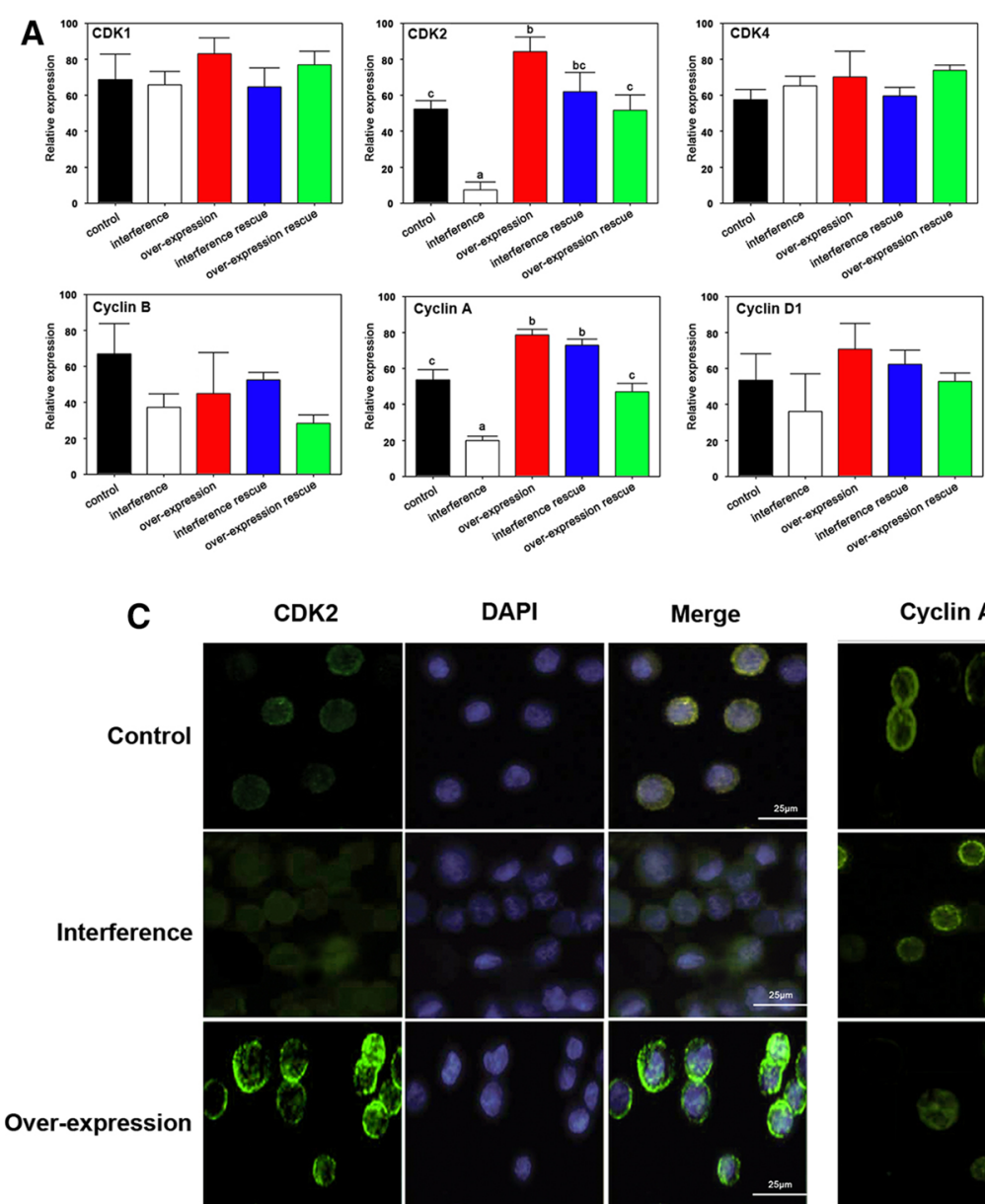

Merge
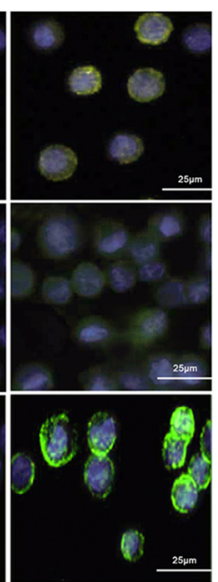

Cyclin A

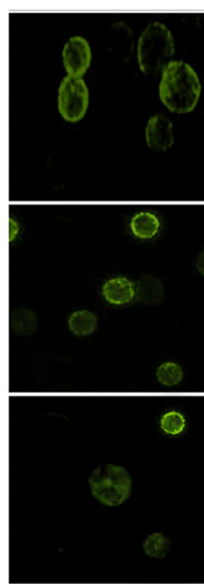

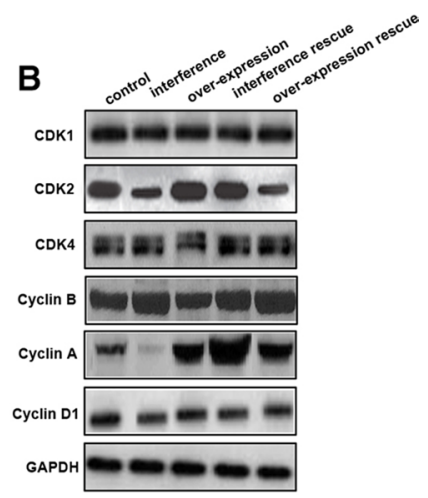

DAPI

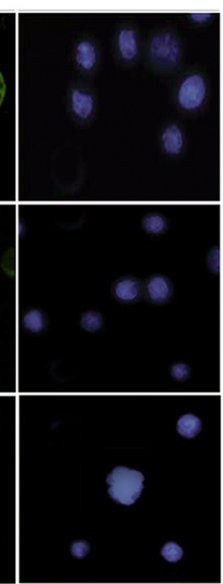

Merge

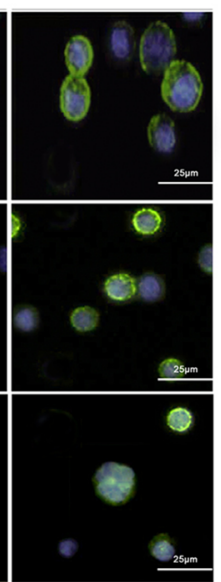

Fig. 4 The effects of PIWIL2 on CDK2 and Cyclin A in NSCLC cells. Real-time PCR (A) and western blot (B) analysis indicated that only CDK2 and Cyclin A is affected by changes in PIWIL2 expression. The characters on the bars represent significant differences between groups (P < 0.05$)$. $\mathbf{C}$ Immunofluorescence analysis of CDK2 and Cyclin A after interference of over-expression of PIWIL2. CDK2 and Cyclin A protein were stained by FITC (green). Nuclei were stained by DAPI (blue)

in 2006 [22, 34], however, little is known regarding the PIWI-piRNA pathway in cancer. Although abnormal PIWI expression resulting in poor prognosis has previously been reported, the specific role of PIWI in cancer cells remains unknown. Additionally, PIWIL2 interference triggers apoptosis, which implies a potential therapeutic strategy for NSCLC.

Because PIWIL2 regulates progression of NSCLC cells by controlling the cell cycle, we quantified cell-cyclerelated proteins affected by PIWIL2. CDK2 and Cyclin A are key factors that control DNA synthesis and the cell cycle [35]; lack of CDK2 and Cyclin A leads to apoptosis [36]. We showed that PIWIL2 promotes expression of CDK2 and Cyclin A both in vitro and in vivo. In a previous study, PIWIL2 could inhibit TGF- $\beta$ signaling via Hsp90 and by promoting T $\beta R$ degradation [37]. Further, PIWIL2 and TGF- $\beta$ expression are negatively correlated
[37]. These findings suggest that PIWIL2 may affect cell proliferation through various means. Our results support previous findings that PIWIL2 promotes cell proliferation.

Over the last decade, evidence has emerged that an epigenetic switch occurs during NSCLC transformation where non-coding RNAs, including miRNAs, piRNAs, and long non-coding RNAs, mediate self-renewal of cancer initiating cells [38]. Lim and colleagues showed that piRNA pathway genes are overexpressed in ovarian cancer [39] and they proposed that PIWIL1 and MAEL inhibit cell invasion. A subsequent study indicated that the RASSF1C-dependent promotion of lung cancer cell proliferation is dependent on IGFBP-5 and PIWIL1, implicating the PIWI-piRNA pathway in tumorigenesis [40]. In vertebrates, abnormal expression of PIWI genes was related to tumorigenesis, sterile and hypogenesis 

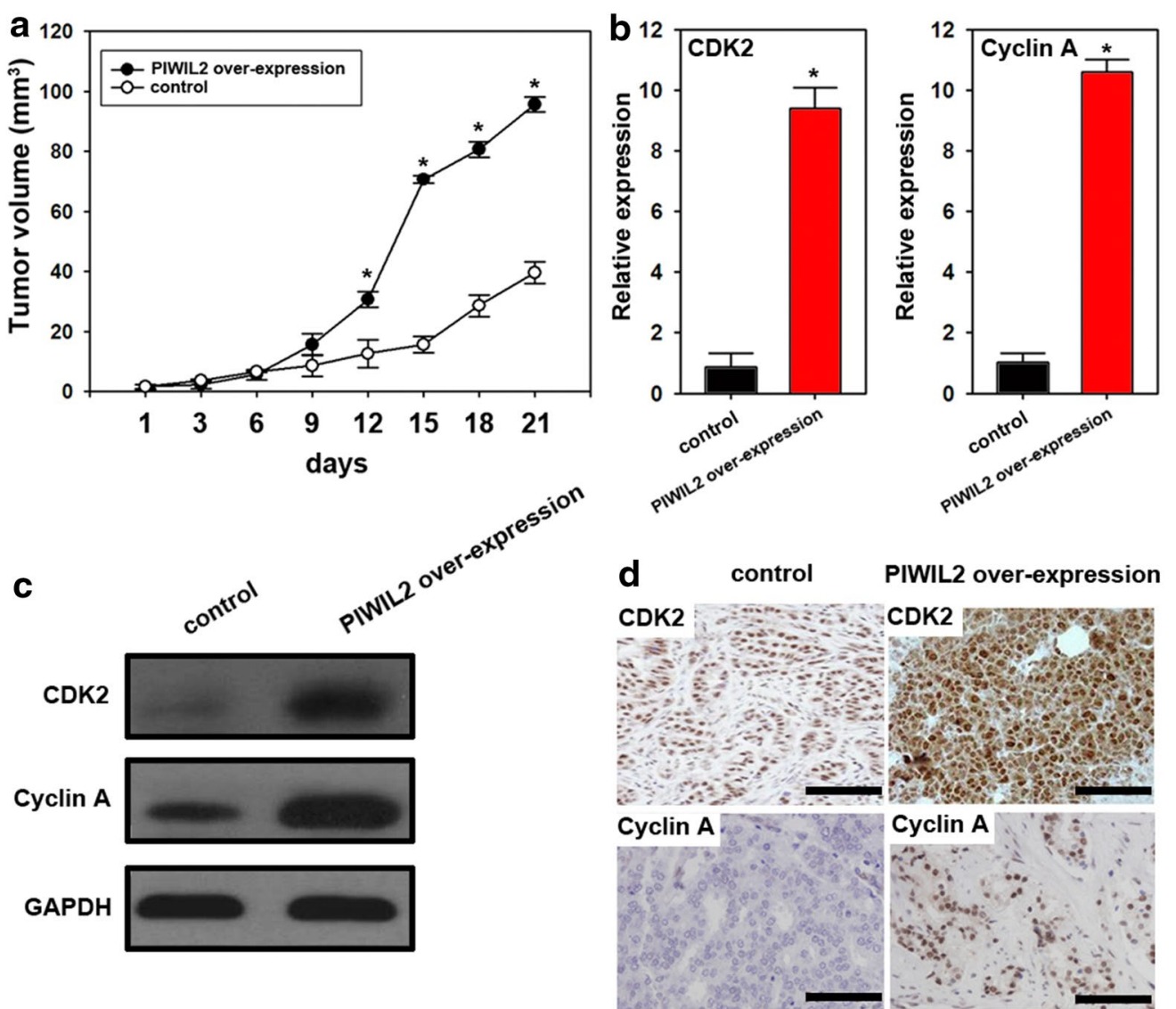

Fig. 5 PIWIL2 promotes tumor growth, and CDK2 and Cyclin A expression in nude mice. a Injection with A549 cells containing the PIWIL2 overexpression vectors promoted tumor growth in a nude mice model $(n=5)$. Real-time PCR $(\mathbf{b})$, western blot $(\mathbf{c})$ and IHC (d) analysis indicated that over-expression of PIWIL2 increased CDK2 and Cyclin A expression in tumor tissues. Asterisk indicates the significant difference between the groups $(\mathrm{P}<0.05)$. For $\mathrm{IHC}$, scale bars $=100 \mu \mathrm{m}$

[41-46]. However, until now, the PIWI-piRNA pathway remains poorly understood. In addition to its role in stabilizing the genome, the PIWI-piRNA pathway is likely involved in tumorigenesis. So far, detailed evidence for the role of PIWI proteins in tumorigenesis remains limited. We showed that PIWIL2 interference inhibited NSCLC progression, both in vitro and in vivo, which suggests potential therapeutic value for PIWIL2 in NSCLC.

\section{Conclusions}

In sum, we showed that increased expression of PIWIL2 is associated with worse prognosis of NSCLC and that PIWIL2 interference could inhibit cell proliferation and activities. Furthermore, CDK2 and Cyclin A were regulated by PIWIL2 both in vitro and in vivo. These findings suggest that PIWIL2 participates in the progression of NSCLC via CDK2 and Cyclin A.

\section{Authors' contributions}

$X Q$ and $J L$ designed the experiment, performed most of the experiments and drafted the manuscript. XZ, XL and QZ carried out the flow cytometric analysis, participated in the design of the study and helped in writing the manuscript. All authors read and approved the final manuscript.

\section{Author details}

${ }^{1}$ The First Affiliated Hospital, China Medical University, NO. 155, Nanjing North Street, Heping District, Shenyang 110001, Liaoning, China. ${ }^{2}$ The Forth Affiliated Hospital, China Medical University, Shenyang, China.

\section{Acknowledgements}

A special thanks to Zhang Zhang for his invaluable assistance in statistical analysis.

Compliance with ethical guidelines

\section{Competing interests}

The authors declare that they have no competing interests.

Received: 28 April 2015 Accepted: 9 September 2015

Published online: 15 September 2015

\section{References}

1. Siegel R, Ma J, Zou Z, Jemal A. Cancer statistics, 2014. CA Cancer J Clin. 2014;64:9-29. 
2. Chang A. Chemotherapy, chemoresistance and the changing treatment landscape for NSCLC. Lung Cancer. 2011;71:3-10.

3. Wroblewski JM, Bixby DL, Borowski C, Yannelli JR. Characterization of human non-small cell lung cancer (NSCLC) cell lines for expression of $\mathrm{MHC}$, co-stimulatory molecules and tumor-associated antigens. Lung Cancer. 2001;33:181-94

4. Rivera MP. Multimodality therapy in the treatment of lung cancer. Semin Respir Crit Care Med. 2004;25 Suppl 1:3-10.

5. Weinstein IB, Joe AK. Mechanisms of disease: oncogene addiction-a rationale for molecular targeting in cancer therapy. Nat Clin Pract Oncol. 2006;3:448-57.

6. Bertolini G, Roz L, Perego P, Tortoreto M, Fontanella E, Gatti L, Pratesi G, Fabbri A, Andriani F, Tinelli S. Highly tumorigenic lung cancer CD133 + cells display stem-like features and are spared by cisplatin treatment. Proc Natl Acad Sci USA. 2009;106:16281-6.

7. Risch A, Plass C. Lung cancer epigenetics and genetics. Int J Cancer. 2008:123:1-7.

8. Juergens RA, Wrangle J, Vendetti FP, Murphy SC, Zhao M, Coleman B, Sebree R, Rodgers K, Hooker CM, Franco N. Combination epigenetic therapy has efficacy in patients with refractory advanced non-small cell lung cancer. Cancer Discov. 2011;1:598-607.

9. Stephens PJ, Greenman CD, Fu B, Yang F, Bignell GR, Mudie LJ, Pleasance $E D$, Lau KW, Beare D, Stebbings LA. Massive genomic rearrangement acquired in a single catastrophic event during cancer development. Cell. 2011;144:27-40.

10. Sharma S, Kelly TK, Jones PA. Epigenetics in cancer. Carcinogenesis. 2010;31:27-36.

11. Kanwal R, Gupta S. Epigenetic modifications in cancer. Clin Genet. 2012:81:303-11.

12. Chénais B. Transposable elements and human cancer: a causal relationship? Biochim Biophys Acta. 2013;1835:28-35.

13. Laird PW, Jaenisch R. The role of DNA methylation in cancer genetics and epigenetics. Annu Rev Genet. 1996:30:441-64.

14. Esteller M, Herman JG. Cancer as an epigenetic disease: DNA methylation and chromatin alterations in human tumours. J Pathol. 2002;196:1-7.

15. Aravin AA, Hannon GJ, Brennecke J. The Piwi-piRNA pathway provides an adaptive defense in the transposon arms race. Science. 2007;318:761-4.

16. Slotkin RK, Martienssen R. Transposable elements and the epigenetic regulation of the genome. Nat Rev Genet. 2007;8:272-85.

17. Siomi MC, Sato K, Pezic D, Aravin AA. PIWl-interacting small RNAs: the vanguard of genome defence. Nat Rev Mol Cell Biol. 2011;12:246-58.

18. Suzuki R, Honda S, Kirino Y. PIWI expression and function in cancer. Front Genet 2012;3:204.

19. Wang D-W, Wang Z-H, Wang L-L, Song Y, Zhang G-Z. Overexpression of hiwi promotes growth of human breast cancer cells. Asian Pac J Cancer Prev. 2013;15:7553-8

20. Wang Y, Liu Y, Shen X, Zhang X, Chen X, Yang C, Gao H. The PIWI protein acts as a predictive marker for human gastric cancer. Int J Clin Exp Pathol. 2012;5:315.

21. Cui L, Lou Y, Zhang X, Zhou H, Deng H, Song H, Yu X, Xiao B, Wang W, Guo J. Detection of circulating tumor cells in peripheral blood from patients with gastric cancer using piRNAs as markers. Clin Biochem. 2011:44:1050-7.

22. Girard A, Sachidanandam R, Hannon GJ, Carmell MA. A germlinespecific class of small RNAs binds mammalian Piwi proteins. Nature. 2006:442:199-202.

23. Qiao D, Zeeman A-M, Deng W, Looijenga L, Lin H. Molecular characterization of hiwi, a human member of the piwi gene family whose overexpression is correlated to seminomas. Oncogene. 2002;21:3988-99.

24. Sasaki T, Shiohama A, Minoshima S, Shimizu N. Identification of eight members of the Argonaute family in the human genome. Genomics. 2003;82:323-30.

25. Liu W, Jiang $X$, Zhang Z. Expression of PSCA, PIWIL1, and TBX2 in endometrial adenocarcinoma. Onkologie. 2010;33:241-5.
26. Lee JH, Jung C, Javadian-Elyaderani P, Schweyer S, Schütte D, Shoukier M, Karimi-Busheri F, Weinfeld M, Rasouli-Nia A, Hengstler JG. Pathways of proliferation and antiapoptosis driven in breast cancer stem cells by stem cell protein piwil2. Cancer Res. 2010;70:4569-79.

27. Liu JJ, Shen R, Chen L, Ye Y, He G, Hua K, Jarjoura D, Nakano T, Ramesh GK, Shapiro CL. Piwil2 is expressed in various stages of breast cancers and has the potential to be used as a novel biomarker. Int J Clin Exp Pathol. 2010;3:328

28. He G, Chen L, Ye Y, Xiao Y, Hua K, Jarjoura D, Nakano T, Barsky SH, Shen $\mathrm{R}$, Gao J-X. Piwil2 expressed in various stages of cervical neoplasia is a potential complementary marker for p16INK4a. Am J Transl Res. 2010;2:156.

29. Li L, Yu C, Gao H, Li Y. Argonaute proteins: potential biomarkers for human colon cancer. BMC Cancer. 2010;10:38.

30. Yao Y, Li C, Zhou X, Zhang Y, Lu Y, Chen J, Zheng X, Tao D, Liu Y, Ma Y. PIWIL2 induces c-Myc expression by interacting with NME2 and regulates c-Myc-mediated tumor cell proliferation. Oncotarget. 2014;5:8466.

31. Fuhrman SA, Lasky LC, Limas C. Prognostic significance of morphologic parameters in renal cell carcinoma. Am J Surg Pathol. 1982;6:655-64.

32. Chapman EJ, Carrington JC. Specialization and evolution of endogenous small RNA pathways. Nat Rev Genet. 2007;8:884-96.

33. Peters $L$, Meister $G$. Argonaute proteins: mediators of RNA silencing. Mol Cell. 2007;26:611-23.

34. Aravin A, Gaidatzis D, Pfeffer S, Lagos-Quintana $M$, Landgraf $P$, lovino $\mathrm{N}$, Morris P, Brownstein MJ, Kuramochi-Miyagawa S, Nakano T. A novel class of small RNAs bind to MILI protein in mouse testes. Nature. 2006:442:203-7.

35. Cardoso MC, Leonhardt H, Nadal-Ginard B. Reversal of terminal differentiation and control of DNA replication: cyclin A and Cdk2 specifically localize at subnuclear sites of DNA replication. Cell. 1993;74:979-92.

36. Katayose Y, Kim M, Rakkar AN, Li Z, Cowan KH, Seth P. Promoting apoptosis: a novel activity associated with the cyclin-dependent kinase inhibitor p27. Cancer Res. 1997:57:5441-5.

37. Zhang K, Lu Y, Yang P, Li C, Sun H, Tao D, Liu Y, Zhang S, Ma Y. HILI inhibits TGF- $\beta$ signaling by interacting with $\mathrm{Hsp} 90$ and promoting T $\beta \mathrm{R}$ degradation. PLoS One. 2012;7:e41973.

38. Costa FF. Non-coding RNAs: new players in eukaryotic biology. Gene. 2005;357:83-94.

39. Lim SL, Ricciardelli C, Oehler MK, Tan IMDA, Russell D, Grützner F. Overexpression of piRNA pathway genes in epithelial ovarian cancer. PLoS One. 2014;9:e99687.

40. Reeves ME, Firek M, Chen S-T, Amaar YG. Evidence that RASSF1C stimulation of lung cancer cell proliferation depends on IGFBP-5 and PIWIL1 Expression levels. PLoS One. 2014;9:e101679.

41. Pan Y, Hu M, Liang H. Wang J-j, Tang L-j: The expression of the PIWI family members miwi and mili in mice testis is negatively affected by estrogen. Cell Tissue Res. 2012;350:177-81.

42. Houwing $S$, Kamminga LM, Berezikov E, Cronembold D, Girard A, van den Elst H, Filippov DV, Blaser H, Raz E, Moens CB, et al. A role for Piwi and piRNAs in germ cell maintenance and transposon silencing in Zebrafish. Cell. 2007;129:69-82.

43. Houwing S, Kamminga L, Berezikov E, Cronembold D, Girard A, Elst H, Filippov D, Blaser H, Raz E, Moens C, et al. A role for Piwi and piRNAs in germ cell maintenance and transposon silencing in Zebrafish. Cell. 2007;129:69-82

44. Kuramochi-Miyagawa S, Kimura T, Ijiri TW, Isobe T, Asada N, Fujita Y, Ikawa M, Iwai N, Okabe M, Deng W. Mili, a mammalian member of piwi family gene, is essential for spermatogenesis. Development. 2004;131:839-49.

45. Zhou Y, Zhong H, Liu S, Yu F, Hu J, Zhang C, Tao M, Liu Y. Elevated expression of Piwi and piRNAs in ovaries of triploid crucian carp. Mol Cell Endocrinol. 2014;383:1-9.

46. Lau NC, Ohsumi T, Borowsky M, Kingston RE, Blower MD. Systematic and single cell analysis of Xenopus Piwi-interacting RNAs and Xiwi. EMBO J. 2009:28:2945-58. 